\title{
Synovitis in SpA—mast cells sail in (despite TNF blockade)
}

Mast cells have a previously unappreciated role in synovial inflammation in spondyloarthritis (SpA), and might be a new therapeutic target in the disease. Reporting in Arthritis \& Rheumatism, Dominique Baeten and colleagues show that these mediators of innate immunity are not only significantly $(P=0.004)$ more abundant in inflamed SpA synovia than equivalent rheumatoid arthritis (RA) synovia, but are also the main cellular source of IL-17 in SpA synovial tissue. Furthermore, mast cell infiltration occurs irrespective of SpA stage or subtype, and is impervious to anti-TNF treatment.

Activation of $\mathrm{B}$ cells and $\mathrm{T}$ cells drives synovitis in RA; in SpA the process is more reliant on cells of the innate immune system, such as macrophages. Nonetheless, mast cells are thought to contribute to synovial inflammation in RA, and have been implicated in joint pathology in the SpA subtype psoriatic SpA.

To investigate further, Baeten and colleagues studied synovial tissue and fluid from 82 patients with SpA and 50 with RA. Psoriatic and nonpsoriatic SpA, established disease, early (untreated) disease, and paired samples (obtained before and after 12 weeks of clinically effective anti-TNF therapy with etanercept) were included. Mast cells were identified by immunohistochemical analysis; their two main functions, degranulation and cytokine production, were probed using quantitative immunofluorescence and ELISA. "Finally," explains Baeten, "we performed functional synovial biopsy explant assays to assess whether mast cell targeting affects synovial inflammation."

\section{4 ...mast cells represented $63 \%$ of IL-17-producing cells... 77}

Besides being more abundant in SpA synovia, mast cells represented $63 \%$ of IL-17-producing cells in the tissue, compared with $26 \%$ in RA samples. Mast cell degranulation seemed minimal in SpA; rather, the production of IL-17 (plus IL-6, IL-8 and probably other inflammatory cytokines that were not investigated) was profoundly affected by mast cell-targeted induction of apoptosis. Other types of IL-17-producing cell, but not IL- $17^{+}$mast cells, were less abundant after etanercept treatment.

Whether mast cell infiltration and production of IL-17 characterizes peripheral SpA, or occurs also in axial disease or extra-articular manifestations, is unknown. Perhaps more important is identifying what drives the influx. "We plan to study the regulation, function and signaling mechanisms of IL-17 family cytokines in mast cells in SpA synovium", says Baeten. The researchers will also investigate crosstalk with stromal cells. As Baeten concludes, understanding these molecular mechanisms "will be the key to designing rational therapies".

Emma Leah

Original article Noordenbos, T. et al. IL-17 positive mast cells contribute to synovial inflammation in spondyloarthritis. Arthritis Rheum. doi:10.1002/art.33396 